\title{
On elastic lateral-torsional buckling analysis of simply supported I-shape beams using Timoshenko's energy method
}

\author{
A.M. Barszcz, M.A. Giżejowski \& Z. Stachura \\ Department of Concrete and Metal Structures, Warsaw University of Technology, Warsaw, Poland
}

\begin{abstract}
The classical energy formulation dedicated to the lateral-torsional buckling of I-section steel members is based on the so-called linear buckling approach (LBA) and inclusion the prebuckling stress resultants in the energy equation. As a result, the buckling analysis may be converted to the linear eigenproblem analysis (LEA). The "exact" closed-form solution of the differential equilibrium equation is obtained only for uniform bending. Moment gradient cases need approximate analytical or numerical methods to be used. Investigations presented in this paper deal with the energy formulation in which the classical energy equation is modified in the way proposed by Timoshenko, so that such an energy approach is named the Timoshenko's energy method. It leads to the nonlinear eigenproblem analysis (NEA). Using this method, practical approximate solutions may be obtained for any asymmetric transverse loading conditions that produce a moment gradient. The stability criterion in this paper is formulated for a general case of transverse loading, the solution of which is obtained by treating the general loading patterns and corresponding moment diagrams as a superposition of symmetric and antisymmetric components. The results are presented in a table format for considered simple loading cases. For combined loading cases, results are presented in the form of nomograms. Solutions obtained for selected combined loading cases are verified with use of LTBeam software.
\end{abstract}

\section{INTRODUCTION}

\subsection{Overview}

The Classical Energy Method (CEM) for the evaluation of elastic beam lateral-torsional buckling problems belongs to the stability theory of thin-walled members. The general formulation of torsion and bending of thin-walled members was developed by Vlasov and summarized in Vlasov (1961). Many monograph publications have then been devoted to stability problems related to torsional or flexural-torsional modes of buckling governed by Vlasov theory, e.g. Březina (1966), Roik et al. (1972), Chen \& Atsuta (1976), Roik (1978), Weiss \& Giżejowski (1991), Trahair (1993), Rykaluk (2012). Energy based methods and differential equilibrium equations were used in the formulation of stability criteria. The energy formulation of the lateral-torsional buckling of beams was studied by many authors, the summary of which has been given by Pi et al. (1992). A way for the improvement of CEM was firstly shown in the monograph of Timoshenko and Gere (1961). In case of LTB problems, energy based solutions may be improved by making use of the minor axis bending differential equilibrium equation. Trahair (1993) refers this method to TEM (Timoshenko's Energy Method) and this terminology is used hereafter. This method was used by many authors to solve different lateraltorsional buckling (LTB) problems of I-shape section beams, e.g. Mohri et al. (2003), Bijak (2015). This study discusses different issues related to the formulation of TEM for the elastic LTB problem of beams in simple and combined loading cases.

DOI: $10.1201 / 9781003132134-8$ 


\subsection{Statement}

Giżejowski et al. (2021) used the classical energy approach to solve the elastic flexuraltorsional buckling problems of beam-columns subjected to a general loading pattern dependent upon a single load parameter $\psi_{i}$ (where $i=M, q, Q$ ), enabling to represent the case of unequal end moments and span loads being different on both half-lengths of the member. When the axial compressive force is equated to zero, the presented there solutions are valid for the lateral-torsional buckling of beams. This paper constitutes research being a continuation of that previously conducted. LTB problems are dealt with showing an improvement of TEM results over those obtained from CEM. In the improved energy method presented in this paper for solving the critical load multiplier of beams, the minor axis secondorder equilibrium equation is used for the evaluation of the second derivative of the minor axis field displacements in order to replacing the classical energy term dependent upon the product of multiplication of $\phi$ (the angle of mean twist rotation that for the present study is equal to the twist rotation $\theta_{x}$ ) and $v^{\prime \prime}$ (second derivative of the minor axis displacement) of the out-of-plane deformation state by the term dependent upon the field twist rotation $\phi$.

\section{LTB OF I-SECTION BEAMS SUBJECTED TO NON-UNIFORM BENDING}

\subsection{Formulation of basic TEM energy equation}

Let us consider a case of the elastic lateral-torsional buckling of simply supported bisymmetric I-section beam of length $L$ subjected to the linear moment gradient being the action effect of unequal end moments and the nonlinear moment gradient being the action effect of span loads $Q_{z, j}$ and $q_{z, i}$ (Figure 1). The classical energy equation is adopted for the linear buckling analysis (LBA) in order to solve the bifurcation load on the elastic primary equilibrium path by using eigenproblem analysis. The Cartesian coordinate system, general loading arrangement and deformation states considered hereafter are shown in Figure 1.

The classical energy equation used in LEA takes the well-known form, e.g. Roik (1978), Trahair (1993):

$$
\begin{array}{r}
\frac{1}{2} \int_{0}^{L}\left\{E I_{z} \delta\left[\left(v^{\prime \prime}\right)^{2}\right]+E I_{w} \delta\left[\left(\phi^{\prime \prime}\right)^{2}\right]+G I_{T} \delta\left[\left(\phi^{\prime}\right)^{2}\right]+2 M_{y} \delta\left(v^{\prime \prime} \phi\right)\right\} d x \\
+\frac{1}{2} \sum_{i} \int_{x_{q 1, i}}^{x_{q 2, i}} q_{z, i} z_{q, i} \delta\left(\phi^{2}\right) d x+\frac{1}{2} \sum_{j} Q_{z, j} z_{Q, j} \delta\left(\phi^{2}\right)=0
\end{array}
$$

where $v=x$-coordinate dependent minor axis displacement; $\phi=x$-coordinate dependent angle of twist rotation; and $I_{z}=$ minor axis moment of inertia; $I_{T}=$ torsion constant; $I_{w}=$

a)

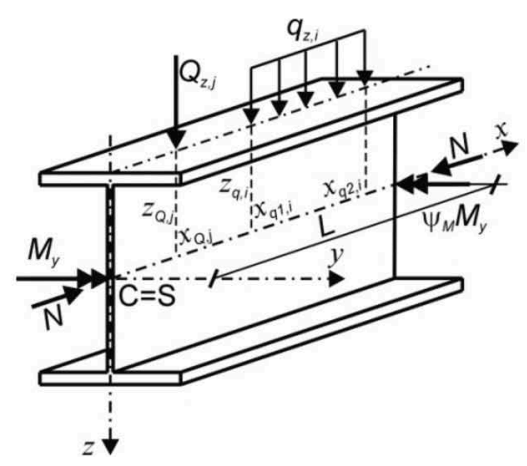

b)

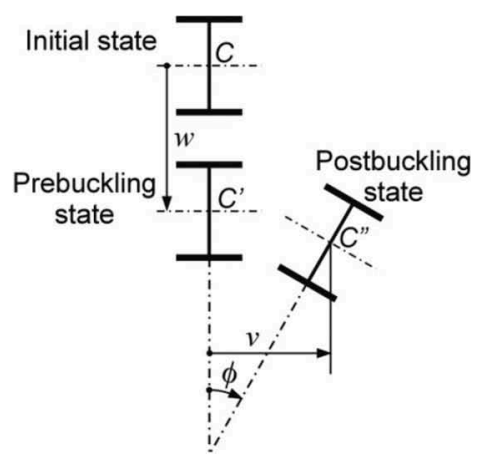

Figure 1. I-section beam: a) considered actions and coordinate system, b) deformation states. 
warping constant; $M_{y}=x$-coordinate dependent major axis moment equation; $E=$ Young's modulus; $G=$ Kirchhoff's modulus; $q_{z, i}=$ component ' $i$ ' of uniformly distributed loads (UDLs); $Q_{z, j}=$ component ' $j$ ' of concentrated loads (CLs), $z_{q, i}, z_{Q, j}=$ section coordinate $z$ of applied UDL and CL.

The minor axis beam differential equilibrium equation corresponding to the minor axis bending and torsion is of the well-known form:

$$
E I_{z} v^{\prime \prime}=-M_{y} \phi
$$

where the section warping free and bending rotation allowed boundary conditions at the member end sections are applied.

Solving Equation (2) for $v^{\prime \prime}$ and substituting to the first and fourth terms of the first integral in Equation (1), a refined form of the energy equation is obtained in which all the terms are dependent upon the angle of twist rotation of the buckling mode and its derivatives. The sum of the first integral terms of Equation (1) involving $v^{\prime \prime}$ and the product of $\phi$ and $v^{\prime \prime}$ becomes:

$$
\frac{1}{2} \int_{0}^{L}\left\{E I_{z} \delta\left[\left(v^{\prime \prime}\right)^{2}\right]+2 M_{y} \delta\left(v^{\prime \prime} \phi\right)\right\} d x=-\frac{1}{2} \int_{0}^{L} \frac{M_{y}^{2}}{E I_{z}} \delta\left(\phi^{2}\right) d x
$$

As a result, the accuracy of the energy solution is uplifted since the approximation of twist rotation is more accurate than that of the product of twist rotation and a guessed curvature of the minor axis deflected shape. Finally, the energy Equation (1) becomes:

$$
\begin{array}{r}
\frac{1}{2} \int_{0}^{L}\left\{E I_{w} \delta\left[\left(\phi^{\prime \prime}\right)^{2}\right]+G I_{T} \delta\left[\left(\phi^{\prime}\right)^{2}\right]-M_{y, \max }^{2} \frac{\left(M_{y} / M_{y, \max }\right)^{2}}{E I_{z}} \delta\left(\phi^{2}\right)\right\} d x \\
+\frac{1}{2} \sum_{i} \int_{x_{q 1, i}}^{x_{q 2, i}} q_{z, i} z_{q, i} \delta\left(\phi^{2}\right) d x+\frac{1}{2} \sum_{j} Q_{z, j} z_{Q, j} \delta\left(\phi^{2}\right)=0
\end{array}
$$

where $M_{y, \max }=$ maximum moment.

For any loading case, the general solution of Equation (4) may be converted to a typical ultimate state criterion used for the codification purposes in the elastic design:

$$
\frac{M_{y, \max }}{M_{c r}}= \pm 1
$$

where $M_{c r}=M_{c r, 0} C_{b c}$ is the critical moment for any major axis moment gradient; $M_{c r, 0}=i_{0} \sqrt{N_{z} N_{T}}$ is the critical moment in the case of uniform major axis bending; $i_{0}=$ polar radius of gyration; $C_{b c}=$ equivalent moment conversion factor for representing the critical moment of any moment gradient case by its uniform moment counterpart.

The following approximations of buckling shape functions were usually adopted for solving CEM buckling problems based on Equation (1):

$$
\begin{gathered}
v=a_{1} \sin (\pi \xi)+a_{2} \sin (2 \pi \xi) \\
\phi=a_{3} \sin (\pi \xi)
\end{gathered}
$$

where $\xi=$ dimensionless coordinate equal to $x / L ; a_{1}, a_{2}$ and $a_{3}=$ unknown buckled shape constants.

Hereafter, Eq. (7) is used and substituted to Eq. (4) in which the terms associated with $q_{z, i}$ and $Q_{z, j}$ are omitted (span loads at the shear centre line). As a result, the stability criterion becomes: 


$$
\delta a_{3} K_{L T} a_{3}=0 \rightarrow K_{L T}=0
$$

in which:

$$
K_{L T}=i_{0}^{2} N_{T}-\frac{M_{y, \max }^{2}}{N_{z}}\left[\left(\frac{M_{y, s, \max }}{M_{y, \max }}\right)^{2} \frac{1}{C_{b s}}+\left(\frac{M_{y, a, \max }}{M_{y, \max }}\right)^{2} \frac{1}{C_{b a}}\right]
$$

where $M_{y, s, \max }=$ maximum moment for the BMD symmetric component; $M_{y, a, \max }=$ maximum moment for the BMD antisymmetric component; $C_{b s}, C_{b a}=$ elemental conversion factors squared for the symmetric and antisymmetric BMDs, the inversions of which are the doubled integrals of the respective products of dimensionless field moment squared and twist rotation shape function squared:

$$
\begin{aligned}
& \frac{1}{C_{b s}}=2 \int_{0}^{1}\left[\frac{M_{y, s}(\xi)}{M_{y, \mathrm{~s}, \max }}\right]^{2} \sin ^{2}(\pi \xi) d \xi \\
& \frac{1}{C_{b a}}=2 \int_{0}^{1}\left[\frac{M_{y, a}(\xi)}{M_{y, a, \max }}\right]^{2} \sin ^{2}(\pi \xi) d \xi
\end{aligned}
$$

Using Equations (8) - (10), the moment conversion factor $C_{b c}$, being denoted by $C_{b c, T E M}$ in the TEM solution, takes the form:

$$
C_{b c, T E M}=\left[\left(\frac{M_{y, s, \max }}{M_{y, \max }}\right)^{2} \frac{1}{C_{b s}}+\left(\frac{M_{y, a, \max }}{M_{y, \max }}\right)^{2} \frac{1}{C_{b a}}\right]^{-\frac{1}{2}}
$$

\subsection{Solutions for simple cases of unequal end moments or unequal half-span loads}

The loading cases considered hereafter are given in Figure 2, namely: unequal end moments (EMs), uniformly distributed loads being unequal in half-lengths (UDLs) and concentrated loads being unequal in half-lengths (CLs).

End moments of different values generate asymmetric non-uniform bending. Let us introduce the moment gradient ratio $\psi_{M}=M_{y M \text {, min }} / M_{y M \text {, max }}$ being a ratio of the minimum end moment divided by the maximum moment (including the sign, cf. Figure 2a). For the case of unequal end moments $C_{b s}=1.00$ and $C_{b a}=7.65$, Equation (11) leads to the following conversion factor:

$$
C_{b c, T E M}=\frac{1}{\sqrt{\left[0.5\left(1+\psi_{M}\right)\right]^{2}+0.1307\left[0.5\left(1-\psi_{M}\right)\right]^{2}}}
$$

a)

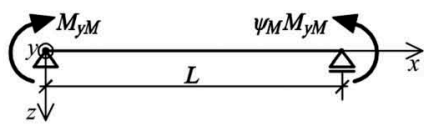

b)

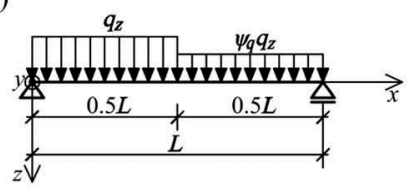

c)

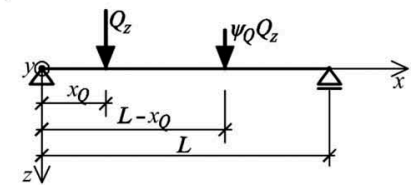

Figure 2. Considered simple loading cases: a) EMs, b) UDLs, c) CLs. 
The solution based on the classical energy method, cf. Equation (1), has been presented first by Trahair (1993) and confirmed by Giżejowski et al. (2021). Recalling $C_{b s}=1.00$ and $C_{b a}=7.71$ from Giżejowski et al (2021), Equation (11) becomes:

$$
C_{b c, C E M}=\frac{1}{\sqrt{\left[0.5\left(1+\psi_{M}\right)\right]^{2}+0.1298\left[0.5\left(1-\psi_{M}\right)\right]^{2}}}
$$

Comparing both solutions one can notice that TEM and CEM lead to the same structure of $C_{b c}$ factor but with different values of $C_{b a}$. The first square root term in the dominator of Equations (12) \& (13) refers to uniform bending while the second one - to antisymmetric bending. It is obvious that the solutions are the same for uniform bending $\left(\psi_{M}=1\right)$ while there is a slight difference for moment gradient cases $\left(-1 \leq \psi_{M}<1\right)$. The TEM solution gives the lesser value of the critical moment. In the case of antisymmetric bending $\left(\psi_{M}=-1\right)$ the difference reaches its maximum value of less than $0.35 \%$ in the critical moment prediction.

The TEM solutions for $C_{b c, T E M}$ concerned with other simple load cases of span uniformly distributed loads in the half-lengths $q_{z, 1}$ and $q_{z, 2} \leq q_{z, 1}$ or concentrated loads $Q_{z, 1}$ and $Q_{z, 2} \leq Q_{z, 1}$ may also be obtained using Equation (11). Factors $C_{b c, T E M}$ for symmetric $\operatorname{BMD}\left(\psi_{i}=1\right)$ and antisymmetric $\operatorname{BMD}\left(\psi_{i}=-1\right)$ are summarized in Table 1 . The results from present study are compared with $C_{b c, C E M}$ obtained by Giżejowski et al. (2021). $\psi_{i}=-1$ means an antisymmetric bending moment diagram $(i=q, Q)$.

Load cases presented in Table 1 generate non-uniform bending. To the best authors' knowledge, the results referred to $C_{b c, T E M}$ for $\psi_{i}=-1$ have not been reported in earlier studies. TEM and CEM solutions for the symmetric load cases coincide with those reported earlier in the literature, $\mathrm{cf}$. references cited at the end of this paper.

\subsection{Solutions for span loads in combination with unequal end moments}

A more important difference between the elastic LTB solutions using classical and refined energy equations may arise for beams under combined loading. The case of combined unequal end moments and UDL is considered hereafter (superposition of loads given in Figure 2a and $2 \mathrm{~b}$ with $\psi_{q}=1$ ). The maximum moment $M_{y \text {, max }}$ within the beam length under unequal end moments and UDL depends upon the moment $M_{y M \text {, } \max }$ - greater of end moments, and $M_{y q \text {, } \max }=0.125 q_{z} L^{2}-$ mid-span moment when the distributed load is acting alone, and upon the moment gradient ratio $\psi_{M}$. Therefore, an additional load effect parameter is introduced $\mu=M_{y q, \text { max }} / M_{y M \text {, max }}$ in order to cover all the load combination cases of unequal end moments and UDL.

Establishing dimensionless moment in Equations (10) and carried out the integration, the coefficients $1 / C_{b s}$ and $1 / C_{b a}$ are obtained, and then by substituting to Equation (11) it allows for the evaluation of the $C_{b c}$ conversion factor related to the combined case of unequal end moments and UDL. The results obtained for different $\mu$ are graphically presented in Figure 3

Table 1. Comparison of TEM and CEM coefficients: $C_{b c, T E M}$ and $C_{b c, C E M}$.

\begin{tabular}{|c|c|c|c|c|c|}
\hline \multirow[b]{3}{*}{ Load type } & \multicolumn{2}{|l|}{ CEM } & \multicolumn{2}{|l|}{ TEM } & \\
\hline & \multicolumn{2}{|c|}{$C_{b c, C E M}{ }^{*}$} & \multicolumn{3}{|c|}{$C_{b c, T E M}{ }^{*}$} \\
\hline & & $\psi_{i}=1$ & $\psi_{i}=-1$ & $\psi_{i}=1$ & $\psi_{i}=-1$ \\
\hline$q_{z}$ & 1.15 & 1.43 & 1.13 & 1.37 & \\
\hline$Q_{z}\left(x_{Q}=L / 2\right)$ & 1.38 & 0.00 & 1.37 & 0.00 & \\
\hline$Q_{z}\left(x_{Q}=L / 3\right)$ & 1.12 & 1.74 & 1.10 & 1.56 & \\
\hline$Q_{z}\left(x_{Q}=L / 4\right)$ & 1.05 & 1.81 & 1.04 & 1.73 & \\
\hline
\end{tabular}


a)

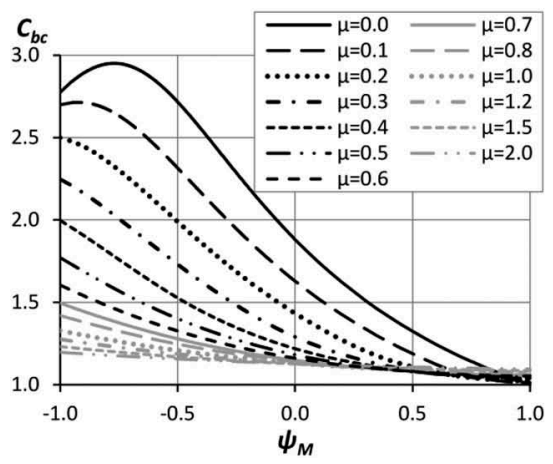

b)

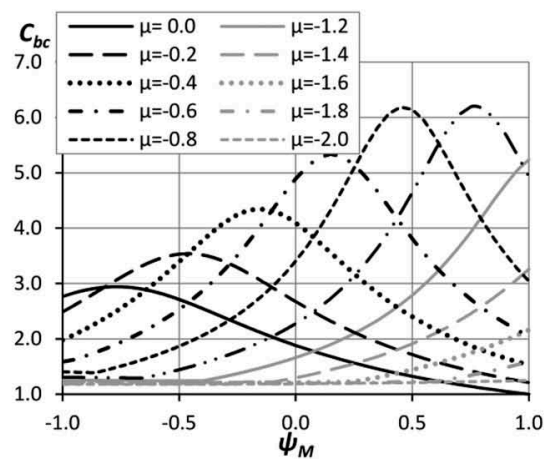

Figure 3. $C_{b c}$ conversion factor for different $\mu$ and $\psi_{M}$ parameters; a) $\mu>0$, b) $\mu<0$.

as a function of $\psi_{M}$. The $C_{b c}$ conversion factor is presented in Figure $3 a$ for $\mu>0$ and in Figure $3 \mathrm{~b}$ for $\mu<0$ in the form of multiple curves dependent upon $\mu$. Nomograms similar to those presented in Figure 3 are included in Annex B of the ECCS Eurocode Design Manual authored by Simoes da Silva et al. (2016).

For positive values of $\mu$, the $C_{b c}$ curves are not continuous since the maximum moment upon the combined loading cases changes its location along the member length as well as because there is a continuous change of the moment diagram shape. For a narrow range of small positive values of $\mu$, the curves go up when $\psi_{M}$ travels from negative towards positive values, reaching the maximum value greater than those for $\psi_{M}=-1$, then they decrease. For larger positive values of $\mu$, the curves decrease from the maximum value reached for $\psi_{M}=-1$.

For negative values of $\mu$, the $C_{b c}$ curves are also not continuous. The curves go up when $\psi_{M}$ travels from negative towards positive values, reaching the maximum value being greater and above those for $\psi_{M}=-1$. The maximum value is slightly above 6 for $\mu$ between -0.6 and -0.8 .

\section{VERIFICATION OF DEVELOPED SOLUTIONS FOR COMBINED LOADING CASES}

Comparing the TEM curves obtained in this study with those of Giżejowski et al. (2021), one may conclude that in some regions of $\mu$ and $\psi_{M}$ the curves presented in Figures $3 \mathrm{a}, \mathrm{b}$ of this paper are importantly different from those yielding from CEM investigations. Generally, the curves from TEM presented in this study are placed lower than those from CEM, especially for high values of $C_{b c}$ appearing in the range of negative $\mu$.

Differences in $C_{b c}$ values for combined loading cases, referred mainly to the range of negative $\mu$ values, need to be verified by finite element numerical simulations and other analytical solutions, especially dedicated for practical applications. In the comparison presented hereafter, the CEM results of Giżejowski et al. (2021) and TEM of present study are verified with use of the finite element simulations made by the LTBeam software available in the public domain. For calculations, IPE300 section is considered for the beam of $6 \mathrm{~m}$ in length, simply supported with regard to bending about both axes and free to warp. The following combined loading cases with unequal end moments are dealt with using $\mu=-1.2$ and shear centre span loads: case 1 of UDL $-\psi_{q}=1$; case 2 of CL at the beam mid-length $-\psi_{Q}=0$. The results of verification are presented in Table 2. Results under a) are from CEM, under b) from TEM and under c) from LTBeam. 
Table 2. Verification of $C_{b c}$.

\begin{tabular}{|c|c|c|c|c|c|c|c|c|}
\hline For $\psi_{M} *$ & & -1.0 & -0.7 & -0.4 & 0 & 0.4 & 0.7 & 1.0 \\
\hline \multirow[t]{3}{*}{ Case 1} & a) & 1.276 & 1.271 & 1.277 & 1.747 & 2.779 & 4.985 & 23.16 \\
\hline & b) & 1.258 & 1.247 & 1.242 & 1.661 & 2.468 & 3.655 & 5.238 \\
\hline & c) & 1.236 & 1.228 & 1.225 & 1.644 & 2.454 & 3.644 & 5.140 \\
\hline \multirow[t]{3}{*}{ Case 2} & a) & 1.309 & 1.386 & 1.670 & 2.580 & 5.575 & 18.36 & 6.376 \\
\hline & b) & 1.263 & 1.317 & 1.543 & 2.175 & 3.277 & 3.957 & 3.421 \\
\hline & c) & 1.244 & 1.300 & 1.528 & 2.165 & 3.250 & 3.834 & 3.309 \\
\hline
\end{tabular}

* Same $\psi_{M}$ values for both combined loading cases considered.

\section{CONCLUSIONS}

The modified energy approach, referred herein to TEM, is discussed in this paper in relation to elastic LTB problems. It is shown that for beam simple load cases CEM and TEM solutions are close to each other while for combined loading cases there might be a noticeable difference. NEA conversion factors $C_{b c, T E M}$ are of smaller values than their LEA counterparts $C_{b c, C E M}$.

Verification of TEM results presented in Table 2 for selected combined loading cases showed that the results obtained in this study are closer to the results from the computer code LTBeam than those yielding from CEM, especially in the range of $\psi_{M}$ between 0.5 and 1.0 (see values being bolded in Table 2).

\section{REFERENCES}

Vlasov, V.Z. 1961. Thin Walled Elastic Beams. Second ed., Jeruzalem: Israel Program for Scientific Translations.

Březina, V. 1966. Stability of metal structures members. Warszawa: Arkady [Polish translation].

Roik, K., Carl, J., Lindner, J. 1972. Biegetorsionsprobleme gerader dünnwandiger Stäbe. Berlin: Wilhelm Ernst \& Sohn.

Roik, K. 1978. Vorlesungen über Stahlbau. Grundlagen. Berlin: Verlag von Wilhelm Ernst \& Sohn.

Chen, W.F. \& Atsuta, T. 1976. Theory of Beam-Columns, vol. 2: Space Behavior and Design, New York: McGraw-Hill.

Weiss, S. \& Giżejowski, M.A. 1991. Stability of metal structures. Rod structures. Warszawa: Arkady [in Polish].

Trahair, N.S. 1993. Flexural-Torsional Buckling of Structures. Boca Raton: CRC Press.

Rykaluk, K. 2012. Stability problems of metal structures, Wrocław: Dolnośląskie Wydawnictwo Edukacyjne [in Polish].

Pi, Y-L, Trahair, N.S. \& Rajasekaran, S. 1992. Energy equation for beam lateral buckling. Journal of Structural Engineering 118(6):1462-1479.

Timoshenko, S.P. \& Gere, J.M. 1991. Theory of Elastic Stability, 2nd edition. New York: McGraw-Hill.

Mohri, F., Brouki, A. \& Roth, J.C. 2003. Theoretical and numerical stability analyses of unrestrained, mono-symmetric thin-walled beams. Journal of Contructional Steel Research 59:63-90.

Bijak, R. 2015. The lateral buckling of simply supported unrestrained bisymetric I-shape beams. Archives of Civil Engineering 61(4): 127-140.

Giżejowski, M.A., Barszcz, A.M. \& Stachura, Z. 2021. Elastic flexural-torsional buckling of steel I-section members unrestrained between end supports. Archives of Civil Engineering 67 (1)[in print].

Simoes da Silva, L., Simoes, R., Gervasio, H. 2016. Design of Steel Structures (2nd Edition). ECCS Mem Matrins: Multicomp Lda. 